\title{
Intelligent Decision Support System for Depression Diagnosis Based on Neuro-fuzzy-CBR Hybrid
}

\author{
Victor E. Ekong ${ }^{1}$, Udoinyang G. Inyang ${ }^{1} \&$ Emmanuel A. Onibere ${ }^{2}$ \\ ${ }^{1}$ Department of Computer Science, Faculty of Science, University of Uyo, Uyo, Nigeria \\ ${ }^{2}$ Department of Computer Science, Faculty of Physical Sciences, University of Benin, Benin City, Nigeria \\ Correspondence: Victor E. Ekong, Department of Computer Science, Faculty of Science, University of Uyo, \\ Akwa Ibom State, Nigeria. Tel: 234-80-5604-3359. E-mail: victor_eshiet_ekong@yahoo.co.uk
}

Received: May 2, $2012 \quad$ Accepted: June 19, $2012 \quad$ Online Published: June 25, 2012

doi:10.5539/mas.v6n7p79 URL: http://dx.doi.org/10.5539/mas.v6n7p79

\begin{abstract}
Depression disorder is common in primary care, but its diagnosis is complex and controversial due to the conflicting, overlapping and confusing nature of the multitude of symptoms, hence the need to retain cases in a case base and reuse effective previous solutions for current cases. This paper proposes a neuro-fuzzy-Case Base Reasoning (CBR) driven decision support system that utilizes solutions to previous cases in assisting physicians in the diagnosis of depression disorder. The system represents depression disorder with 25 symptoms grouped into five categories. Fuzzy logic provided a means for handling imprecise symptoms. Local similarity between the input cases and retrieved cases was achieved using the absolute deviation as the distance metric, while adaptive neuro-fuzzy inference system handled fuzzy rules whose antecedents are the mapped local similarities of each category of symptoms for global similarity measurement, upon which the retrieved cases are ranked. The 5 best matched cases are subjected to the emotional filter of the system for diagnostic decision making. This approach derives strengths from the hybridization since the tools are complementary to one another.
\end{abstract}

Keywords: depression disorder, neuro-fuzzy, decision support, similarity measure, depression diagnosis

\section{Introduction}

Depression is a common mental disorder in primary health care, but its diagnosis and effective treatment is controversial because of variations in clinical practice, lack of evidence on the best treatments and access to them, and because of its multifaceted nature and contested meaning of symptoms (Dowrick, 2004). Depressive symptoms range along a continuum from every day sadness, loss of interest to suicidal depression.

Although many other symptoms occur in varying combinations, the illness is a causal factor in many chronic conditions such as diabetes, cardiovascular diseases, HIV/AIDS resulting in higher costs to the healthcare system (Maja, Meifania, \& Tharam, 2008; World Health Organization (WHO), 2009; Kessler, 2002). The recognition and treatment of depression is a challenging area of clinical practice, especially in primary care where there are many patients with various presentations and a multitude of causes for distress (Lester \& Howe, 2008). In depression diagnosis, physicians utilize a number of cognitive behavioural therapy (CBT) assessment tools such as Becks depression inventory (BDI) and Hamilton's Rating Scale for Depression (HRSD) or the Montgomery-Asberg Depression Rating Scale (MADRS) to establish severity levels of the disease in order to determine therapy (Ariyanti, Kusumadewi, \& Paputungan, 2010; Nameroff, 2006). Classification of the disease is therefore based on the patients' subjective description of symptoms and the physician's judgment as to whether they meet the criteria of the diagnostic and statistical manual of mental disorders, version 4 (DSM-IV) (American Psychiatric Association (APA), 1994). Diagnosis of the disorder by primary care physicians is difficult due to the complexity and confusing nature of the disease (Mondimore, 2006; Mila, Kielan, \& Michalak, 2009).

Medical diagnosis has undergone different phases of research from mathematical and statistical approaches which are mostly engaged to enhance the quality of medical data estimations, to Artificial Intelligent (AI) approaches. The inadequacy of statistical estimation techniques is that quality cannot be guaranteed when dealing with incomplete, noisy and non-linear data (Antoni, Jorge, \& Paulo, 2008). AI approaches provides reasoning capability, which consists of inference from facts and rules using heuristics, pattern matching or other 
search approaches. Recent developments in medical diagnostics has embraced the AI approaches such as genetic algorithms (GA), neural networks (NN), Fuzzy logic (FL), rule based systems (RBS) and Case Based Reasoning (CBR) to develop tools for diagnosis and for predicting treatment responses (Wan, Wan, \& Fadzilah, 2006).

In this work, AI techniques of neural networks, fuzzy logic and CBR are combined to model a DSS for the diagnosis of depression disorders. NNs are constructed to imitate the intelligent human biological processes of learning, self-modification and adaptation. Although NN is good at handling non-linear, noisy or incomplete data it has very weak explanation mechanism which is highly desirable in medical decision support systems (MDSS) (Uzoka, Obot, \& Baker, 2009). Fuzzy logic provides a means for dealing with imprecision, vagueness and uncertainties in medical data (Zadeh, 1965). Neuro-fuzzy inference systems provide self-learning intelligent systems that are capable of handling uncertainties in a diagnosis process (Jang, Sun, \& Mitzutani, 1997). CBR entails the use of a set of concrete past situations, called cases, stored in a knowledge base referred to as a case base to solve a new problem (Aamodt, 1994). Combinations of CBR with other intelligent methods have been explored for more effective knowledge representation and problem solving. Hybrid CBR systems are reported in (Obot, Akinyokun, \& Udoh, 2008; Prentzas, \& Harzilygeroudis, 2009; Lopez-Fernandez, FdezRiverola, Rboiro-Jato, Glez-Pena, \& Mendez, 2011; Begum \& Mema, 2011). Among the hybrid CBR systems, a neuro-fuzzy CBR is not widely reported; the closest are combinations of FL with CBR or NN with CBR in the implementation of medical diagnosis systems. Hence, the contribution to knowledge of this work stems not only from the hybridization of NN, FL and CBR but also the fusion of neuro-fuzzy inference system and fuzzy similarity matching in the implementation of a MDSS.

\section{Related Works}

In Marks et al., (2004), a computer-aided CBT (CCBT) is described for delivering selfhelp for sufferers with phobic, panic, anxiety, obsessive-compulsive and depressive disorders. Although the approach greatly reduced the demand on therapist's time, it lacked the patient - doctor feedback mechanism which is crucial for depression therapy. In Razzouk, Mari, Wainer, \& Sigulem (2006), a pilot study for modeling the diagnoses of schizophrenia and other psychotic disorders is reported. In Mila et al. (2009), a fuzzy semiotic framework for modeling imprecision in the assessment of depression is presented. In Begum, Ahmed, Funk, Xiong \& Scheele (2009), a case based DSS is developed for individual stress diagnosis using finger temperature profiles. The approach enabled the reuse of experience from previous cases with analyzed temperature and stress profiles. In Suhasini, Palanivel, \& Ramalingam (2010), an ANN approach is described for diagnosing depression using radial basis function (RBF) and back propagation neural networks. The approach showed great promise in accurately identifying the psychiatric problem among patients, but it lacked an explanation mechanism. In Ariyanti et al. (2010), a BDI version 2 test assessments is reported that utilizes a fuzzy inference system to represent all the factors of BDI-II's 21 questions.

\section{Method}

In our methodology, we propose an intelligent system that incorporates ANN, FL and CBR in the diagnosis of depression. The procedure of hybrid platforms design described in (Shanthi, Sahoo, \& Saravanan, 2009; Ajith, 2005; Medsker, 1999; Fuller, 1995) are studied and modified to suit the design. The block diagram for the proposed neural network, fuzzy logic and CBR hybrid is presented in Figure 1.

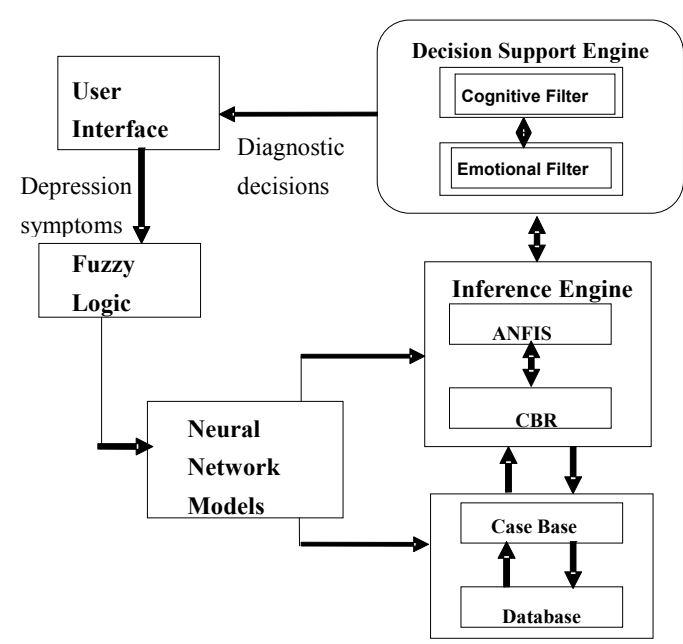

Figure 1. Block diagram of depression diagnosis IDSS hybrid platform 


\subsection{Fuzzification Subsystem}

As shown in Figure 1, the neural network receives fuzzified symptoms of depression as inputs. The fuzzy set of depression symptoms $\left(\mathrm{S}_{\mathrm{i}, \mathrm{j}}\right)$ and depression diagnosis $\left(\mathrm{R}_{\mathrm{i}}\right)$ are $\{$ low, medium, high, very high $\}$ and $\{$ Near Absent, Mild, Moderate, Severe\} respectively. $\mathrm{S}_{\mathrm{i}, \mathrm{j}}$ is the $j$ th symptom of theith patient, $\mathrm{R}_{\mathrm{i}}$ represents the result of depression diagnosis of theith patient. The $21 \mathrm{BDI}-\mathrm{II}$ set of parameters for depression disorder provided in Becks (1996) and Physiological parameters described in Hildrum, Mykletun, Holman and Dahl (2008) are adopted for this work. The 21 BDI-II symptoms are grouped based on Wan, Hu, Moore and Ashford (2008) categorization of Becks cognitive model of depression. A summary of depression disorder attributes and their categories is presented in Table 1.

Table 1. Attributes of depression disorder

\begin{tabular}{cccc}
\hline SN & Category & Patients' attributes & Code \\
\hline 1 & & Sadness & SAD \\
2 & & Crying & CRY \\
3 & Emotional & Irritability & IRR \\
4 & factor (A) & Loss of interest & LOI \\
5 & & Agitation & AGI \\
\hline 6 & & Self criticalness & SCR \\
7 & & Indecision & IND \\
8 & & Self dislike & SDL \\
9 & & Worthlessness & WOT \\
10 & Cognitive & Pessimism & PES \\
11 & factors (B) & Guilty feelings & GUI \\
12 & & Concentration difficulty & CON \\
13 & & Past failure & FAL \\
14 & & Punishment feelings & PUN \\
\hline 15 & & Tiredness & TRD \\
16 & Physical & Changing sleep patterns & CSL \\
17 & factors (C) & Change in appetite & CAP \\
18 & & Loss of interest in sex & LSX \\
\hline 19 & & Loss of pleasure & LOP \\
20 & Motivational & Loss of energy & LOE \\
21 & factors (D) & Suicidal thoughts & SUI \\
\hline 22 & & Age & AGE \\
23 & Physiological & Diastolic blood pressure & DBP \\
24 & factor (E) & Systolic blood pressure & SBP \\
25 & & Body mass index & BMI \\
\hline & & &
\end{tabular}

A fuzzy set is represented by expressing it as a function and then map the elements of the set to their degree of membership. Some of the membership functions that exist are Gaussian, Triangular, Trapezoidal, S-function and L-function (Vijaya, Nehemiah, Kannan, \& Bhuvaneswari, 2010). Triangular and Trapezoidal membership functions have been used extensively due to their computational efficiency (Jang et al., 1997). The work of Dubois \& Prade (1996) guided the choice of the triangular membership function for this work and gives the general form of the triangular membership function in Equation (1)

$$
\mu(x)= \begin{cases}1, & \text { if } x=b \\ \frac{x-a}{b-a} & \text { if } a \leq x<b \\ \frac{c-x}{c-b} & \text { if } b \leq x<c \\ 0, & \text { if } c \leq x\end{cases}
$$


where a and $\mathrm{c}$ are the parameters governing triangular membership functions; $b$ represents the value for which $\mu(\mathrm{x})=1$ and is defined as $b=\frac{(a+c)}{2}$. The actual membership functions of each element in the fuzzy set are derived as described in (Jang et al., 1997; Akinyokun, Obot, \& Uzoka, 2009). Examples includeLow $(A)=$ $f(\mathrm{~A},[0.10,0.25,0.40])$ and $\operatorname{Severe}(\mathrm{R})=f(\mathrm{R},[0.47,0.74,1.0])$.

\subsection{Training Subsystem}

The fuzzified symptoms serve as inputs to the neural network models. Each Neural network is a two layered feed forward architecture which interacts with each other as shown in Figure 2.

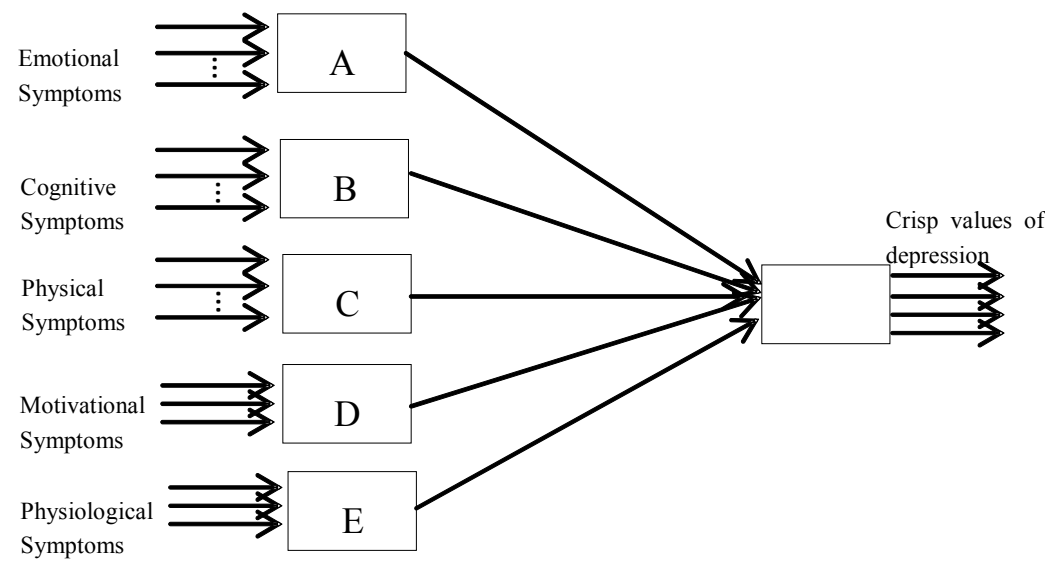

Figure 2. Block diagram of NN for depression DSS

The algorithm used to train the networks is the back-propagation algorithm with sigmoid function for hidden and output layer neurons' transformation. The neuro-fuzzy architecture is presented in Figure 3. In Figure 3, the NN trained by the subsystem consists of 25 nodes in the symptoms layer; each node represents a linguistic value in the fuzzy set of depression symptoms. The hidden layer has 5 nodes, each node correspond to a category of depression attributes considered in this work. The output layer consists of 4 nodes, each representing the level of severity of depression disorder.

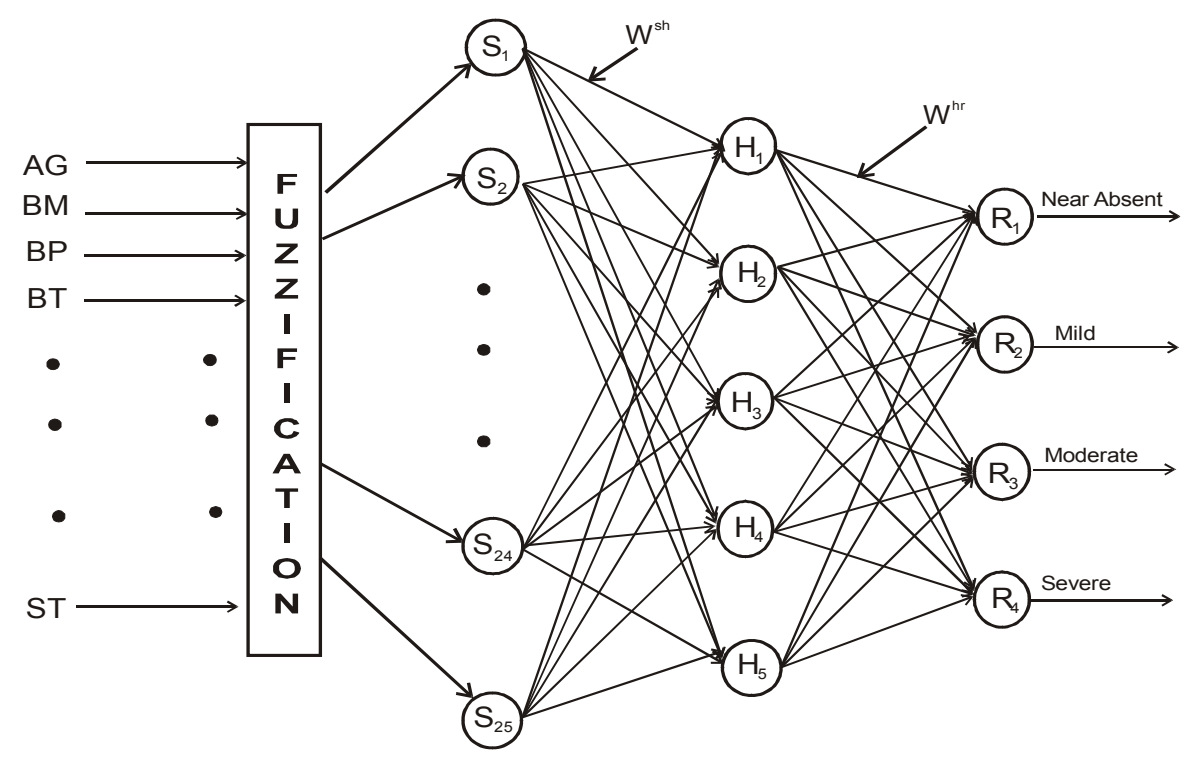

Figure 3. Neuro-Fuzzy Architecture of IDSS depression disorder diagnosis 
Let $p, q$ and $t$ represent the number of neurons in the input, hidden and output layers respectively. $W^{\text {sh }}$ represents the weight vector between symptoms (input) layer nodes to hidden layer node. $W^{h r}$ represents the weight vector between hidden layer nodes to output layer node. $A^{r}$ represents the expected output for a given patient data. For each patient's data $S_{i, j}\left(s_{1}, s_{2}, \ldots s_{p}\right)$, in the training set, respective weights are computed using Equations (2) to (6). The output of the input layer neurons is derived from Equation (2), the input to the hidden layer neurons are derived from Equation (3) while outputs of the hidden layer neurons are computed from Equation (4). Input to the output layer neurons and output of the output layer neurons are represented in Equations (5) and (6) respectively.

$$
\begin{aligned}
O_{j}^{i} & =x_{j}, j=1,2, \ldots, p \\
S_{k}^{h} & =\sum_{j=1}^{p} O_{j}^{i} W_{j k}^{s h}, k=1,2, \ldots, q \\
O_{k}^{h} & =\frac{1}{1+e^{-S_{k}^{h}}}, k=1,2, \ldots, q \\
S_{m}^{r} & =\sum_{k=1}^{q} O_{k}^{h} W_{k m}^{h r}, m=1,2, \ldots, t \\
O_{m}^{r} & =\frac{1}{1+e^{-S_{m}^{r}}}, m=1,2, \ldots, t
\end{aligned}
$$

\subsection{Inference Subsystem}

In the inference subsystem, the mapping from an input (new case) to the retrieved case (target) is measured using fuzzy similarity matching and adaptive neuro-fuzzy inference system (ANFIS). The results from the fuzzy similarity matching are the parameters driving the ANFIS which provides results from which decisions are made. We denote cases in the case base as $Z \times w$ matrices, where $w$ represents the categories of depression symptoms and $\mathrm{z}$ the number of patients cases in the case base. Depression diagnostic cases are in a column vector. Let A and B be patients' symptom and diagnostic cases respectively.

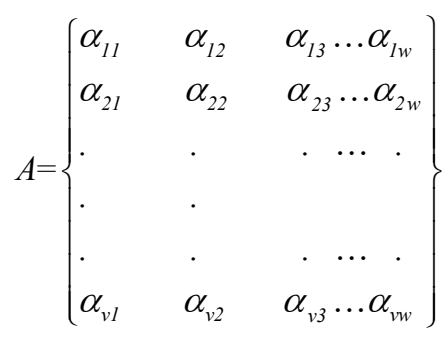

In this work, we set $\mathrm{w}=5$; the ith row vector, $\mathrm{A}_{\mathrm{i}, \mathrm{u}}=\left[\mathrm{a}_{\mathrm{i} 1}, \mathrm{a}_{\mathrm{i} 2}, \ldots \mathrm{a}_{\mathrm{i}, 5}\right]$ represents the ith patient's case, where $\mathrm{u}=1,2,3,4,5, \mathrm{a}_{\mathrm{i}, \mathrm{u}}$ is the result of the actual mapping of the linguistic value to the crisp value within the degree of membership. We therefore have the row vector as in Equation (8).

$$
A=\left[a_{j, u}\right]_{u=1}^{5}
$$

Let $\mathrm{E}$ and $\mathrm{T}$ represent the transpose of the row vectors of new cases (input) and retrieved cases (target) respectively. Let $\mathrm{L}$ be the distance metric matrix; $l_{i, j}=l\left(e_{i}, t_{j}\right)$ be the distance between the $i t h$ input element and $j$ th target element. There are $y \times y$ possible distance metrics; where $\mathrm{y}$ is the total number of symptoms in a category, in this work, we adopt the absolute deviation in Equation (9) as the distance metric. The general form of Matrix L is as shown in Equation (10).

$$
l\left(e_{i}, t_{j}\right)=\left|e_{i}-t_{j}\right|
$$

We denote the various distance metrics matrices as $\mathrm{L}_{\mathrm{A}}, \mathrm{L}_{\mathrm{B}}, \mathrm{L}_{\mathrm{C}}, \mathrm{L}_{\mathrm{D}}$, and $\mathrm{L}_{\mathrm{E}}$. Each column of $\mathrm{L}$ represents the absolute deviation of a symptom from other of symptoms in a particular category. Let $\alpha_{\mathrm{i}, \mathrm{j}}$ be the elements of $\mathrm{L}$, $\delta_{i, j}(i=1,2, \ldots, \mathrm{y})$ denotes the average absolute deviation of symptom $j$ from $i$ other symptoms in a particular category; it is given in Equation (11) 


$$
\mathcal{L}\left\{\begin{array}{cccc}
\left|e_{1}-t_{1}\right| & \left|e_{1}-t_{2}\right| & \ldots & \left|e_{1}-t_{y}\right| \\
\left|e_{2}-t_{1}\right| & \left|e_{2}-t_{2}\right| & \ldots & \left|e_{2}-t_{y}\right| \\
\left|e_{3}-t_{1}\right| & \left|e_{3}-t_{2}\right| & \ldots & \left|e_{3}-t_{y}\right| \\
\cdot & \cdot & \cdots & \cdot \\
\cdot & \cdot & \cdots & \cdot \\
\cdot & \cdot & \cdots & \cdot \\
\left|e_{y}-t_{1}\right| & \left|e_{5}-t_{2}\right| & \cdots & \left|e_{5}-t_{y}\right| \\
5 & \\
\sum_{i, j}=\frac{\sum_{i=1}^{y} \alpha_{i j}}{y}
\end{array}\right.
$$

The normalized absolute deviation, $\left\|\delta_{k}\right\|$ of the $k t h$ category is the local similarity measure;

$$
\begin{gathered}
\left\|\delta_{k}\right\|=\frac{\delta_{i j}}{\frac{1}{y}\left(\sum_{j=1}^{y} \delta_{i j}\right)} \\
\left\|\delta_{k}\right\|=\frac{\frac{\sum_{i=1}^{y} \alpha_{i j}}{y}}{\frac{1}{y}\left(\sum_{j=1}^{y} \frac{\sum_{i=1}^{y} \alpha_{i j}}{y}\right)}
\end{gathered}
$$

The fuzzy set \{very close, close, slightly close\} is used to represent the local similarities between the input case and target case and its membership function is defined as follows;

$$
S_{L(k)}= \begin{cases}\text { "Very Close" } & \text { if }\left\|\delta_{k}\right\|<0.35 \\ \text { "Close" } & \text { if } 0.35<\left\|\delta_{k}\right\| \leq 0.65 \\ \text { "Slightly Close" } & \text { if }\left\|\delta_{k}\right\|>0.65\end{cases}
$$

The global similarity measure $\left(\mathrm{S}_{\mathrm{g}}\right)$ is derived from the fuzzy rule of the local similarity measure. Example of such rules is:

If ( $S_{L(1)}$ is 'Very Close') and ( $S_{L(2)}$ is 'Close') and ... ( $S_{L(y)}$ is 'Slightly Close') then $S_{g(i)}$ is 'Very Similar'

From the rule set, the training pattern $\left\{\left(\left\|\delta_{I}\right\|, S_{L(1)}\right),\left(\left\|\delta_{2}\right\|, S_{L(2)}\right), \ldots,\left(\left\|\delta_{y}\right\|, S_{L(y)}\right)\right\}$ is the general form of the antecedent part while an element in the Fuzzy set, $\mathrm{S}_{\mathrm{g}}=\{$ Very similar, Similar, Slightly Similar $\}$ is the fuzzy target output. The training patterns are evaluated by the ANFIS. The fact that Mamdani's Fuzzy Inference is intuitive, has widespread acceptance and well suited for human cognition, informed its choice as the inference mechanism (Chai, Jia, \& Zhang, 2009). The architecture of the ANFIS is presented in Figure 4. It is a Multiple Input, Single Output (MISO) architecture with five layers as in (Mendel, 2001; Inyang \& Inyang, 2011). Out of the five layers, the first and fourth layers consist of adaptive nodes while the second, third, and fifth layers consist of fixed nodes. The input layer (Layer 0) has 5 nodes, each corresponding to a category of depression symptoms; they pass external crisp value to layer 1 . Layer 1 consists of 15 fuzzification nodes; the outputs of this layer are the fuzzy membership grade defined by:

$$
O_{1, i}=\mu_{A_{i}}\left(x_{1}\right), \text { for } i=1,2,3 \text {; }
$$




$$
\begin{aligned}
& O_{1, i}=\mu_{B_{i-3}}\left(x_{2}\right), \text { for } i=4,5,6 ; \\
& O_{1, i}=\mu_{C_{i-3}}\left(x_{3}\right), \text { for } i=7,8,9 ; \\
& O_{1, i}=\mu_{D_{i-3}}\left(x_{4}\right), \text { for } i=10,11,12 ; \\
& O_{1, i}=\mu_{E_{i-3}}\left(x_{5}\right), \text { for } i=13,14,15 .
\end{aligned}
$$

The triangular membership function in Equation (1) such that $\mathrm{a} \leq x<\mathrm{b}$ is adopted. These outputs serve as the inputs of Layer 2. Layer 2 is the antecedent rules layer, it provides the firing strength of the rules with and operator as the T-norm. The output is given as follows:

$$
\begin{gathered}
O_{2, i}=\omega_{i}=\mu_{A_{i}}\left(x_{1}\right) \times \mu_{B_{i}}\left(x_{2}\right) \times \mu_{C_{i}}\left(x_{3}\right) \times \mu_{D_{i}}\left(x_{4}\right) \times \mu_{E_{i}}\left(x_{5}\right), i=1,2,3,4,5 \\
\omega_{i}=\operatorname{Min}\left\{\mu_{A_{i}}\left(x_{1}\right) \times \mu_{B_{i}}\left(x_{2}\right) \times \mu_{C_{i}}\left(x_{3}\right) \times \mu_{D_{i}}\left(x_{4}\right) \times \mu_{E_{i}}\left(x_{5}\right)\right\}
\end{gathered}
$$

The output of the normalization layer, (Layer 3) is given in Equation (22)

$$
\begin{gathered}
\varpi_{i}=\frac{\omega_{i}}{\omega_{i}+\omega_{2}+\omega_{3}+\omega_{4}+\omega_{5}}, i=1,2,3,4,5 \\
\varpi_{i}=\frac{\omega_{i}}{\sum_{i=1}^{5} \omega_{i}}
\end{gathered}
$$

The product of each rule's normalized firing level $\left(\varpi_{i}\right)$ and the rule's output $\left(\mathrm{S}_{i}^{g}\right)$ is represented in layer 4 . For the $i$ th node

$$
\mathrm{R}_{\mathrm{i}}{ }^{=} \varpi_{i} \mathrm{~S}_{i}^{g}\left(\omega_{i}\right) \quad \text { where } i=1,2,3, \ldots, 243
$$

The aggregation (sum) neuron is layer 5 , it computes the overall system output as the sum of all incoming signals as given in Equation (25), which represent the global similarity match of the input case and a retrieved case.

$$
S=\sum_{i=1}^{243} \varpi_{i} S_{i}^{g}\left(\omega_{i}\right)
$$




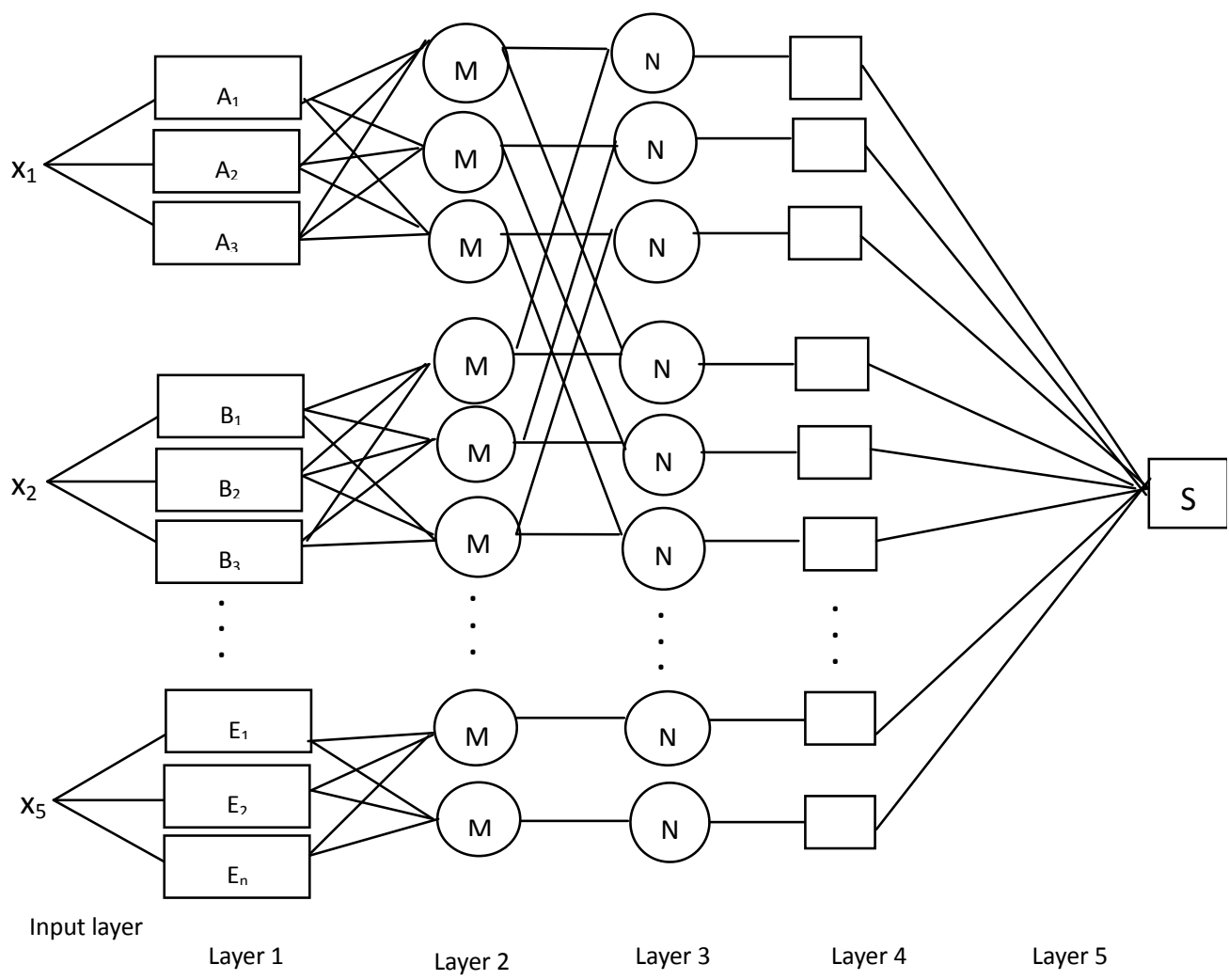

Figure 4. ANFIS Architecture for IDSS hybrid

The result of the ANFIS for all the cases in the case base are ranked and evaluated by the decision support engine. The best 5 matches are selected by the cognitive filter and presented for analysis by the emotional filter. Some factors that may be considered by the emotional filter may include treatment resistance, suicide risk, hospitalization status and so on. The diagnostic decisions for the best matched case is used by the physician for the current case thereafter updated and retained as a new solved case in the case base.

\section{Conclusion and Future Work}

We have presented a neuro-fuzzy CBR model as a decision support system for the diagnosis of depression based on overall severity of symptoms. The proposed hybrid system framework introduces a similarity matching driven neuro-fuzzy architecture that provides flexibility for physicians measuring the severity levels of symptoms and symptoms' category. The fuzzified local similarities of symptoms' categories form the decision variables evaluated by the Mamdani-type ANFIS whose crisp output represent the global similarity between the query case and target case. In this work we have also proposed CBR and neuro-fuzzy hybrid framework for solving real life problems. In our future work, we intend to implement the model in an environment characterized by Windows 7 operating system, Microsoft Access database management system, MatLab and Java programming languages and to evaluate the performance of the system based on standard performance metrics. The use of a hybrid intelligent system undoubtedly offers superior benefit in terms of performance and usability over solitary intelligent systems. This work therefore, proposes an approach for diagnosing depression disorder which will also be extended to quantify the severity levels of other depression related dysfunctions like diabetes, cardiac, liver, lungs and cancer diseases.

\section{References}

Aamodt, A. (1994). Case Based Reasoning: Fundamental issues, Methodological Variations and System Approaches. AI Communications, 7, 39-59.

Ajith, A. (2005). Nature and Scope of AI Techniques. Handbook of Measuring System Design, Peter, H. S. \& Richard, T. (Eds). USA: John Wiley \& Sons, Ltd. 
Akinyokun, O. C., Obot, O., \& Uzoka, F. M. E. (2009). Application of Neuro-Fuzzy Technology in Medical Diagnosis: Case Study of Heart Failure. In Proceeding of World Congress on Medical Physics and Biomedical Engineering, 25(12), 301-304.

American Psychiatric Association. (1994). Diagnostic and Statistical Manual for mental disorders $\left(4^{\text {th }}\right.$ ed.). Washington DC.

Antonio, D., Jorge, H., \& Paulo, D. C. (2008). Neural, Fuzzy and Neurofuzzy System for Medical Applications. In Intelligent and adaptive Systems in Medicine, Series in Medical physics and Biomedicine Engineering, Olivier C. L. H. \& Keith J. B. (Eds.). USA: Taylor Francis group, CRC press, 127-170.

Ariyanti, R. D., Kusumadewi, S., \&Paputungan, I. V. (2010). Beck Depression Inventory Test Assessment Using Fuzzy Inference System. In Proceeding of International Conference on Intelligent Systems Modeling and Simulation, IEEE Computer Society, 6-9. http://dx.doi.org/10.1109/ISMS.2010.12.

Beck, A. T. (1996). Comparison of Beck Depression Inventories - IA and II in Psychiatric Outpatients. Journal of Personality Assessment, 67(3), 588-597. http://dx.doi.org/10.1207/s15327752jpa6703_13

Begum, S., \& Mema, D. (2011). Fuzzy Algorithms for Pattern Recognition in Medical Diagnosis. Assam University Journal of Science \& Tech., 7(7), 1-12.

Begum, S., Ahmed, M. U, Funk, P., Xiong, N., \& Scheele, B. V. (2009). A Case-Based Decision Support System for Individual Stress Diagnosis Using Fuzzy Similarity Matching. Journal of Computational Intelligence, 25(3), 180-195. http://dx.doi.org/10.1111/j.1467-8640.2009.00337.x

Chai, Y., Jia, L., \& Zhang, Z. (2009). Mamdani Model Based Adaptive Neural Inference System and its Application. World Academy of Science, Engineering and Technology, 51, 845-852.

Dowrick, C. J. (2004). Beyond Depression: A New Approach to Understanding and Management. London: Oxford Medical Publications, Oxford University Press.

Dubois, D., \& Prade, H. (1996). What are Fuzzy Rules and How to Use Them? Fuzzy Sets Systems, 84(2), 169-185. http://dx.doi.org/10.1016/0165-0114(96)00066-8

Fuller, R. (1995). Neural Fuzzy Systems. Lecture notes delivered at Turku Centre for Computer Science (TUCS), University of Turku, Finland.

Hildrum, B., Mykletun, A., Holman, J., \& Dahl, A. (2008). Effect of Anxiety and Depression on Blood Pressure: 11 Year Longitudinal Study. British Journal of Psychiatry, 193, 108-113. http://dx.doi.org/10.1192/bjp.bp107.045013

Inyang, U. G., \& Inyang, M. U. (2011). A Neuro-Fuzzy Decision Support Framework for Tenants Satisfaction Assessment in Residential Properties. World Journal of Applied Science and Technology, 3(1), 146-154.

Jang, J. S. R., Sun, C. T., \& Mitzutani, E. (1997). Neuro-Fuzzy and Soft Computing: A Computational Approach to Learning and Machine Intelligence. Upper Saddle River, USA: Prentice Hall Inc., pp. 8-33.

Kessler, R. C. (2002). Epidemiology of depression. In L. H. Gotlib \& C. L. Hamman (Eds) Hand Book of Depression. New York: Guilford Press.

Lester, H., \& Howe, A. (2008). Depression in Primary Care: Three Key Challenges. Postgrad Medical Journal, 84, 545-548. http://dx.doi.org/10.1136/pgmj.2008.068387

Lopez-Fernandez, H., Fdez-Riverola, F., Reboiro-Jato, M., Glez-Pena, D., \& Mendez, J. R. (2011). Using CBR as Design Methodology for Developing Adaptable Decision Support Systems. Efficient Decision Support Systems Practice and Challenges from Current to future, Chiang J., (Ed.), USA: Intech Publishers, 123-126.

Maja, H., Meifania, C., \& Tharam, S. D. (2008). Towards Mental Health Ontology, Proceedings of IEEE Int'l Conference on Bioinformatics and Biomedicine, p. 284.

Marks, I., Kenwright, M., McDonough, M., Whittaker, M., O’Brien, T., \& Mataix-Cols, D. (2004). Saving Clinicians' Time by Delegating Routine Aspects of Therapy to a Computer: A Randomised Controlled trial $\begin{array}{llll}\text { in Panic/Phobia disorder. Psychological Medicine, } & \text { 34, }\end{array}$ http://dx.doi.org/10.1017/S003329170300878X

Medsker, L. R. (1999). Hybrid Intelligent Systems, Kluwer Academic Publishers ( $2^{\text {nd }}$ printing). Boston, MA, USA. 
Mendel, J. (2001). Uncertain Rule-Based Fuzzy Inference Systems: Introduction and New Directions. Prentice-Hall, PTR, USA.

Mila, K., Kielan, K., \& Michalak, K., (2009). A Fuzzy Semiotic Framework for Modeling Imprecision in the Assessment of Depression. IFSA_EUSFLAT2009, 1717-1722.

Mondimore, F. (2006). Depression, The Mood Disease. Baltimore: John Hopkins University Press, 2715 North Charles St., 3-37.

Nemeroff, C. B. (2006). The Burden of Severe Depression: A Review of Diagnostic Challenges and Treatment Alternatives. Journal of Psychiatric Research. http://dx.doi.org/10.1016/j.jpsychires.2006.05.008

Obot, O. U., Akinyokun, O. C., \& Udoh, S. S. (2008). Application of Soft Computing Methodologies in the management of Hypertension. Journal of Computer Science and its Applications, 15(2), 131-147.

Prentzas, J., \& Harzilygeroudis, I. (2009).Combination of Case-Based Reasoning with Other Intelligent Methods. International Journal of Hybrid Intelligent systems, 6(2809), 189-209. http://dx.doi.org/10.3223/IOS-2609.

Razzouk, D., Mari, J., Wainer, J., \& Sigulem, D. (2006). Decision Support System for the Diagnosis of Schizophrenia Disorders. Brazilian Journal of Medical and Biological research, 39(1), 119-128.http://dx.doi.org/10.1590/S0100-879X2006000100014

Shanthi, D., Sahoo, G., \& Saravanan, N. (2009). Evolving Connection Weights of Artificial Neural Networks Using Genetic Algorithm with Application to the Prediction of Stroke Disease. Medwell International Journal of Soft Computing, 4(2), 95-102.

Suhasini, A., Palanivel, S., \& Ramalingam, V. (2010). Multi Decision Support for Psychiatry Problems. International Journal of Computer Application, 1(25), 74-84.

Uzoka, F. M. E., Obot, O., \& Baker, K. (2009).A performance comparison of fuzzy logic and AHP as engines for the development of intelligent medical diagnosis system. In Proc. of $9^{\text {th }}$ Int'l Conf. of Nigeria Computer Society (NCS), (MacGlobe'09), Abuja,20, 135.

Vijaya, K., Nehemiah, H. K., Kannan, A., \& Bhuvaneswari, N. G. (2010). Fuzzy Neuro Genetic Approach for Predicting the Risk of Cardiovascular Diseases. International Journal of Data Mining, Modeling and Management, 2(4), 388-402. http://dx.doi.org/10.1504/IJDMMM.2010.035565

Wan, H., Wan, I., \& Fadzilah, S. (2006). Artificial Intelligence in Medicine. Journal of Health Informatics. Retrieved from http://hi-europe.info/files/2002/9980.htm

Wan, J., Hu, B., Moore, P., \& Ashford, R. (2008). Intelligent mobile computing in the treatment of depression. 3rd International Conference on Pervasive Computing and Applications, ICPCA 2008, 6-8 October 2008, Alexandria, Egypt, 650-655.

World Health Organization. (2009). Mental Health: Depression. Retrieved from http://www.who.int/mental_health/management/depression/definition/en

Zadeh, L. A. (1965). Fuzzy sets. Information and Control, 8, 338-353. http://dx.doi.org/10.1016/S0019-9958(65)90241-X 